\title{
PERCEPCIÓN DE LA CALIDAD DE LOS SERVICIOS EN LA CARRERA INGENIERÍA DE EMPRESAS MODALIDAD DUAL
}

PERCEPTION OF QUALITY IN THE CAREER OF ENGINEERING BUSSINESS - DUAL MODE

\section{CÉSAR FREIRE', GABRIELA HURTADO², KARINA GOVEA ${ }^{3}$}

\footnotetext{
Universidad Católica de Santiago de Guayaquil. cesarfreire@cu.ucsg.edu.ec

2 Universidad Católica de Santiago de Guayaquil. gabriela.hurtado@cu.ucsg.edu.ec

3 Universidad Católica de Santiago de Guayaquil. karina.govea@cu.ucsg.edu.ec
}

\begin{abstract}
RESUMEN
Esta investigación con referencia a la homogeneidad de la percepción de los servicios que brinda la carrera de Ingeniería de Empresas - Modalidad Dual de la Universidad Católica Santiago de Guayaquil, basada en tres aspectos considerados críticos por los estudiantes: (a) Servicios generales de la carrera, (b) Servicios docentes y (c) Servicios administrativos. La investigación se desarrolló sobre la base de un análisis estadístico no paramétrico para poder determinar la homogeneidad de las percepciones de calidad por niveles, lo cual permitió validar los resultados globales a partir de las obtenidas. Como resultado se tuvo que la percepción de la calidad de los servicios que brinda la carrera basada en los tres niveles son iguales. Los niveles de satisfacción global y satisfacción docente mostraron una igualdad en la percepción de las diversas cohortes, mientras que el nivel administrativo mostró dos subgrupos con diferentes percepciones de calidad.
\end{abstract}

PALABRAS CLaVE: satisfacción de la calidad universitaria, prueba no paramétrica, homogeneidad de percepciones.
ABSTRACT This paper deals with a research based on the homogeneity of the perception of the services provided by the career of Engineering Business - Dual Mode in at the Catholic University Santiago de Guayaquil, considering three aspects established as critical by the students: (a) general career services, (b) teachers services (c) administrative services. There search was focused on a non parametric statistical analysis to determine the homogeneity in terms of students perceptions about the quality levels in order to confirm the obtained results. As a result, the perception of the quality of the services provided by the race based on the three levels is equal. Levels of overall satisfaction and satisfaction showed teaching equality in the perception of the various cohorts while the administrative level showed two subgroups with different perceptions of quality.

KEYWORDS: satisfaction of university quality, non-parametric tests, homogeneity perceptions. 
INTRODUCCIÓN

Según Hierro (1994), etimológicamente la palabra "universidad" deriva de universitas, la cual significa: conjunto de los elementos constitutivos de una colectividad. Históricamente sele usó para caracterizar los gremios, las corporaciones y las fraternidades de personas dedicadas al mismo oficio o a la búsqueda de un fin común. En estas agrupaciones con base en la finalidad común, se distinguía cuidadosamente entre los maestros y los aprendices: los magistri y los discipuli. El conjunto de los maestros y discípulos constituyó la universitasmagistrorum et scholarium que poseía privilegios y fueros propios.

La universidad es el fruto de una serie de tradiciones históricas, entre ellas: a) La griega, de la cual recibió el espíritu de libre discusión, así como "su misión de libre investigación"; b) Del mundo latino germano adopta la aplicación práctica de la cultura, que creó la universidad que comprende las escuelas profesionales que son los planteles que están destinados a la investigación científica y que vienen a unirse a los colegios, dedicados a la investigación y creación de una cultura general; c) A medida que la sociedad se fue democratizando, la universidad adquiere como aspecto la difusión cultural. A partir de la cual hoy día, la universidad se ha convertido en el centro de la conservación, creación y difusión de la cultura para todos los ámbitos de la realidad social, es decir, que la hace llegar hasta los grandes grupos sociales que existen, los cuales han sido separados de la universidad por razones económicas.

Es así que desde la aparición de la globalización y la descentralización de las economías, la educación en todos sus niveles debe ser considerada como un bien público. En este sentido, debe albergar a todos los actores de la sociedad, catapultando en la cima al individuo como ser humano y a los motores que dinamizan las economías y las empresas. La sinergia en la concepción del diseño curricular que se pone de manifiesto entre la formación universitaria y las áreas estratégicas de las diferentes empresas se ha convertido actualmente en un elemento indispensable en el marco teórico-práctico de profesionales exitosos. Este proceso se estructura en un contexto donde convergen los diferentes saberes de las aulas, la practicidad requerida en un mundo empresarial competitivo y la vanguardia de nuevas aplicaciones que mejoren su posicionamiento y su rentabilidad.

La universidad contemporánea debe enfrentarse a desafíos para poder desempeñar su rol como educadora, formadora de los jóvenes talentos en los diferentes campos y dominios de las disciplinas. Esto ha de conducirlo de cara a retos profesionales en un entorno empresarial, dinámico y vanguardista. Entre ellos se destacan la incorporación de determinados parámetros para la gestión educativa: (a) Calidad académica, (b) Sistema de gestión de calidad en los procesos (c) Currículos armonizados con las necesidades del entorno empresarial en áreas estratégicas de las organizaciones, (d) Interacción y compromiso del medio empresarial con la formación de los futuros profesionales, (e) $\mathrm{Mo}^{-}$ vilidad estudiantil, y (f) Participación en redes académicas. Con el propósito de enfrentar estos nuevos desafíos es fundamental que la universidad, en sinergia con los diferentes actores de la sociedad, procure la internacionalización del conocimiento, la inclusión de metodologías innovadoras de estudio y desarrollo tecnológico a través de las Tecnologías de la Información y las Comunicaciones (TIC).

Según Dieter (2013), la Modalidad Dual, creada por empresarios alemanes en 1974, se ha convertido en un modelo educativo que permite la interacción didáctica y sistematizada de dos ambientes de aprendizaje: el aula y la empresa; que se complementan mediante actividades coordinadas y evaluadas a lo largo de su desarrollo. Vega (2005) la define como una modalidad de formación profesional, y por ende educativa, que realiza su proceso de enseñanza-aprendizaje en dos lugares distintos, en una institución educativa donde realiza actividades teóricoprácticas y en una organización donde ejecuta actividades didáctico-productivas.

Según Araya (2008), el principio fundamental de este modelo pedagógico corresponde a la relación educación-trabajo en la formación profesional, que se cimienta en los enfoques tecnológico y humanista y debe ser abordado desde perspectivas filosóficas para determinar el tipo de ciudadano que se desea formar, sus valores universales como individuo y a partir de su convivencia con otros. Desde el fundamento epistemológico define el saber y las diversas modalidades de conocimiento, su fundamento psicopedagógico comprende un proceso de enseñanza y de aprendizaje que interrelaciona la psicología y la pedagogía para orientar esa actividad en dos lugares distintos, la universidad y la empresa, mediante un enlace cooperativo, el fundamento socioeconómico que establece la visión de formación del recurso para la productividad y que se apega al desarrollo económico 
y social del individuo y de la sociedad en la que está inmersa.

Este proceso formativo integra varios procesos, el primero de ellos inicia en la concepción del diseño curricular, posteriormente interviene la formación en fase teórica, la misma que se ejecuta a través de tutorías académicas integradas, trabajos autónomos y otros varios componentes propios del aprendizaje colaborativo y asertivo; siendo el componente práctico fundamental en el proceso de formación. El tercero corresponde a la fase práctica, la misma que se ejecuta en empresas formadoras de todos los sectores de la producción y que se lleva a cabo en la realización y aplicación de una serie de actividades sistematizadas en diferentes áreas estratégicas de las organizaciones; tales como mercadotecnia y ventas, producción y logística, costos y finanzas y talento humano. En el período de fase práctica o denominada también período de prácticas preprofesionales, la elaboración de un proyecto empresarial en cada una de las áreas estratégicas se constituye en el principal producto de este aprendizaje; puesto que este implica el mejoramiento, rediseño, innovación o restructuración de las mismas, aportando así al mejoramiento de la competitividad de la organización.

\section{ANTECEDENTES}

En este contexto interactivo entre la universidad y empresas, las teorías del aprendizaje con sus diferentes significados, han ejercido una notable influencia en los procesos de enseñanza-aprendizaje desde la década de los cuarenta del pasado siglo, con la aparición de diferentes expertos que analizaban las formas de aprender relacionadas al comportamiento de los seres humanos, y al conocimiento, entre otros factores. Entre las más importantes se mencionan la teoría del constructivismo y del cognitivismo, de las cuales se pueden extraer postulados diferentes. Estas, al unirse pueden también llegar a formar nuevas corrientes y tendencias que son viables y aplicables en la modalidad dual desde la educación superior.

La metodología de la Modalidad Dual plantea un conocimiento dinámico y enriquecido de paralelismo didáctico que es el resultado de la sinergia de los escenarios: aula y empresa.

Estos escenarios son transformados en los ambientes de aprendizaje, permitiendo en los estudiantes, el desarrollo de competencias por medio de los resultados de aprendizaje, diseña- dos para cada nivel a lo largo de su formación universitaria.

De esta manera, la Modalidad Dual demanda que el proceso de aprendizaje se centre en un tipo de estudiante activo y en sus estructuras cognitivas previas sobre las cuales se ha adquirido conocimiento; puesto que es necesario transferir la teoría a la práctica de manera real en cada uno de los semestres de estudio. Estos conocimientos previos pueden ser mejorados y adaptados al entorno, es decir a la realidad organizacional de cada empresa formadora, a la naturaleza del negocio y a sus condiciones inherentes a su misión, visión, cultura empresarial, etc., así como también a las condiciones culturales, al aporte del lenguaje en el acceso al nuevo conocimiento. Si bien también pueden relacionarse a la motivación intrínseca del sujeto que aprende, esta en algunos casos no es manipulable ya que depende de su propio interés, generando así aprendizaje significativo puesto que los nuevos conocimientos se toman a la luz de los previos.

Según Hernández (2002) deacuerdoalabordaje del aprendizaje significativo, esta modalidad de estudio por su ambiente dinámico, flexible, contemporáneo y competitivo requiere que el aprendizaje permita la elaboración de mapas conceptuales, de esquemas y otras herramientas pedagógicas que lo validen como significativo. Es necesario plasmar lo que se logró interiorizar de manera sistemática y resumida de forma que genere aportes a las empresas formadoras, en cualquiera de las áreas en las que se esté desarrollando (mercadotecnia y ventas, producción y logística, costos y finanzas y talento humano).

Es así, que el docente de la modalidad dual, el tutor académico y el instructor empresarial se convierten en una guía para el estudiante, en un mediador, en el sujeto que plantea a los estudiantes conflictos para los cuales deben encontrarse soluciones creativas e innovadoras que provoquen un impacto positivo durante el proceso de aprendizaje. El mediador debe permanecer también en constante aprendizaje y la empresa formadora debe convertirse en el entorno donde se han producido estas nuevas construcciones.

Este proceso incluye el uso de las TIC, como herramienta primordial para la interiorización de los fundamentos teóricos, de las particularidades de los procesos de formación práctica y el andamiaje de las redes del conocimiento, 
a partir de la aplicación de simulaciones, teorías de juegos, programas, blogs, wikis, etc. (Salinas, 2004). Es relevante para la modalidad dual la elaboración de proyectos, sean éstos individuales o grupales, tanto en la fase teórica en el aula como en la fase práctica en las empresas formadoras, en las cuales se deben conjugar el saber, el saber hacer y el saber ser. Es decir, lo conceptual, lo procedimental y lo actitudinal. Entonces, el estudiante puede construir su propio conocimiento sirviéndose de los entornos, de los grupos colaborativos, del uso de los métodos de inducción y deducción en el que los nativos digitales del siglo XXI aplican las herramientas tecnológicas para aprender, para conceptualizar ideas, para generar y construir nuevo conocimiento.

En conclusión, la teoría del constructivismo por sus tendencias y constructos es absolutamente aplicable a la modalidad dual en las instituciones de educación superior. Esta postura obedece a que centra al estudiante como protagonista del proceso, como constructor, codificador y decodificador del conocimiento, como el autor del aprendizaje significativo en esquemas de colaboración, de trabajo en equipo en relación al entorno en el que se desenvuelve, como sujeto dispuesto, entrenado teórica y prácticamente para aprender haciendo.

Han existido diversos estudios en relación a la medición de la percepción de la calidad en el ámbito universitario, y se justifican en tratar de brindar un servicio basado en estándares que generen un retorno adecuado a nivel educativo. Ruano (2002) enfoca la importancia en poder desarrollar preguntas dirigidas al nivel de calidad, de tal manera que garanticen la obtención de resultados. Realiza un análisis considerando factores relacionados a la docencia, renovación curricular y desempeño profesional. Si consideramos diversos niveles en la medición de la calidad se puede garantizar cobertura en los resultados. Es necesario poder realizar levantamientos de información considerando varios bloques para poder obtener datos más específicos y de mejor utilidad.

Uno de los aspectos más importantes es la calidad del docente, según Merida (2006) es importante que este construya su identidad a partir de su posicionamiento en el proceso de generación del conocimiento. Esto valida la necesidad de considerar al docente como un componente de prioridad en la percepción de la calidad estudiantil, dado que se convierte en un actor directo en la formación del estudiante.
La innovación en los procesos universitarios genera eficiencia y por ende incide en la calidad. Numerosos estudios presentan el impacto de la innovación de la docencia en la educación superior, y cómo se deben considerar ciertos factores prioritarios para mejorar los niveles de enseñanza a nivel docente y la calidad (Mauri, T., Coll, C., y Onrubia, J., 2004).

Existen diversas metodologías y modelos áulicos referidos al proceso de aprendizaje. La innovación en los procesos puede influir sobre la percepción de los estudiantes. "Actualmente nuestra sociedad del conocimiento precisa de estructuras organizativas flexibles en la educación superior" (Hinojo, Aznar y Cáceres, 2009, p. 2). Es importante considerar la percepción estudiantil a nivel universitario para poder generar cambios y realizar planes de mejora a nivel universitario. Existen diversas maneras de influir en los niveles de mejora académica, tanto a nivel administrativo como docente, para impactar en el grado de recepción del aprendizaje por parte de los estudiantes. Hay estudios que tratan de medir de manera más directa la percepción en relación a la calidad universitaria, pero previo a medir la percepción hay que definir correctamente aquellos factores sobre los cuales será medida la calidad en cuanto al servicio docente que brindan las universidades.

El rendimiento universitario depende de la calidad de la educación superior que se brinde es por eso que este componente debe estar presente en las organizaciones educativas. "Es de interés considerar que el análisis del rendimiento académico de los estudiantes universitarios mediante la investigación, constituye un factor imprescindible en los debates en torno a la búsqueda de la calidad de la educación superior» (Garbanzo, 2007, p. 19).

Para una adecuada medición de la calidad de la educación es necesario generar escalas de medición que capturen la percepción de los estudiantes en cuanto al servicio educativo. Con el objeto de tener una visión completa de los requerimientos estudiantiles, han de ser considerados factores como la capacidad para proveer el servicio, comprensión de necesidades y los elementos tangibles (Ríos, 2005). Diversos escenarios de instrucción superior son evaluados en términos de calidad a partir de las percepciones de los estudiantes universitarios. Este proceso evaluador descubre la relación entre el contexto de enseñanza-aprendizaje y el rendimiento estudiantil. Por contexto se entiende la forma de brindar el conocimiento que involucra procesos 
de calidad según De la Fuente, J., Martínez, J., Peralta, F., \& García, A. (2010).

La enseñanza universitaria debe manejarse a través de estándares. Este proceso involucra a los docentes. La gestión administrativa debe autocriticarse con el fin de tomar medidas correctivas que permitan mejorar los escenarios de enseñanza a nivel superior. Las percepciones a nivel docente son prioritarias tal y como lo indican Coromina Rovira, E., Tesouro, M., Capell Castañer, D., Teixidó Saballs, J., Pèlach Busom, J., y Cortada, R. (2006), quienes evaluaron la percepción ante la incorporación de competencias genéricas y encontraron que existe una gran aceptación por parte de los docentes en la mejora de sus competencias genéricas.

Los estudiantes de la carrera de Ingeniería de Empresas de la Modalidad Dual perciben niveles considerables de la calidad universitaria bajo tres componentes debidamente definidos, sin embargo es necesario demostrar si existen diferencias significativas en los diversos niveles o cohortes a los que representan los alumnos, por lo tanto se puede validar la investigación bajo las siguientes hipótesis:

- H1: La percepción de la calidad de la carrera Ingeniería de Empresas - Modalidad Dual en términos generales es homogéneo en todos los niveles lectivos.

- H2: La percepción de la calidad del servicio administrativo en la carrera Ingeniería de Empresas - Modalidad Dual es homogéneo en todos los niveles lectivos.

- H3: La percepción de la calidad de los docentes en la carrera Ingeniería de Empresas - Modalidad Dual es homogéneo en todos los niveles lectivos.

Existen investigaciones que han demostrado igualdad de medias bajo criterios no paramétricos, tal es el caso de Lema Soto, L., Salazar Torres, I. C., Varela Arevalo, M. T., Tamayo, J., Rubio, A., \& Botero, A. (2009) quienes realizan un proceso de muestreo al cual someten a una prueba de igualdad de medias a través de pruebas no paramétricas para poder evidenciar la significancia de la igualdad en cuanto al nivel de percepciones. Así mismo se han realizado estudios referentes a estrategias de aprendizaje universitario en los que se aplicaron técnicas no paramétricas obteniendo resultados alentadores, según Herrera, L., y Lorenzo - Quiles, O. (2009) "El análisis realizado a través de la prueba Kruskal-Wallis, para determinar si el lugar y las condiciones de estudios varían en función del curso en el que se encuentran los alumnos, mostró que no existían diferencias significativas en ninguno de los ítems" (p. 6).

\section{METODOLOGÍA}

Para la recolección de la información se utilizó una encuesta, la cual fue aplicada a 65 estudiantes, considerando un nivel de confianza del 95\% y un margen de error más estricto que el 5\% considerando técnicas de población finita:

$$
n=\frac{N * Z^{2} * p * q}{e^{2 *(N-1)+z^{2} * p * q}}(1)
$$

Bajo esta premisa se realizó el método de estratificación de la muestra en base a la proporción de la población haciendo uso de técnicas de aleatoriedad, la cual permitió levantar ciertos criterios en cuanto a percepciones de calidad. Se utilizó la escala de Likert para tratar de medir la percepción bajo cinco niveles. Se capturó la percepción bajo el componente de satisfacción general de la carrera, del docente y de los servicios administrativos específicamente. Los tópicos que se involucraban eran niveles y subniveles relacionados a: satisfacción global de la carrera, b: satisfacción docente, y c: Satisfacción administrativa.

Según Lind, D., Marchal, W., y Wathen, S. (2012), la prueba de Kruskal-Wallis es paramétrica eficiente que permite probar igualdad de medias y considerando que los datos están a escala ordinal, y bajo el supuesto de que las poblaciones no siguen una distribución normal, se utilizó la prueba no paramétrica propuesta por Kruskal, W., \& Wallis, W. A. (1952) que solo requiere datos de nivel ordinal.

El objeto de la aplicación de la prueba permitió validar si existen diferencias significativas en la percepción de la calidad entre las diferentes cohortes de la carrera de Ingeniería de Empresas. El estadístico de prueba a utilizarse en la investigación es:

$$
K=(N-1) \frac{\sum_{i=1}^{g} n_{i}\left(\bar{r}_{i}-\bar{r}\right)^{2}}{\sum_{i=1}^{g} \sum_{j=1}^{n_{i}}\left(r_{i j}-\bar{r}\right)^{2}}(2)
$$

Donde $\mathrm{K}$ representa al estadístico de prueba de Kruskall-Wallis, $g$ el número de poblaciones, ni es el número de observaciones en el grupo $i, r_{i j}$ es el rango entre todas las observaciones $j$ del grupo $i$, y $n$ es el número de total de observaciones entre todos los grupos. 
Para poder determinar el comportamiento de promedio de $r_{i}$, se procedió a aplicar el siguiente comportamiento.

$$
\bar{r}_{i}=\frac{\left(\sum_{i=1}^{n_{i}} r_{i j}\right)}{n_{i}}(3)
$$

Y para estimar la media de todos los rij

$$
\bar{r}=\frac{(N+1)}{2}(4)
$$

De tal manera que el resultado del estadístico KW con un nivel de significancia del 5\% permitió probar la igualdad entre los grupos involucrados.

Dada la alta dispersión en los datos se optó por realizar un contraste múltiple de rangos para la satisfacción en cuanto a servicios administrativos, de tal manera que se utilizó un total de $n$ ciclos como observaciones $n_{j}$, la media muestral estimada $\bar{y}_{j}$, para poder establecer diferencias entre las dos medias muestrales de tal manera que:

$$
\widehat{\Delta}_{j_{1} j_{2}}=\bar{y}_{j_{1}}-\bar{y}_{j_{2}}(5)
$$

Por lo que se procede a estimar las comparaciones múltiples considerando $M$ constante del proceso elegido LSD, de tal manera que:

$$
M=t_{\propto / 2, n-q}(6)
$$

El método LSD exige normalidad de los datos, por lo que se lo evidenció con las aplicaciones en subgrupos de sesgo y curtosis estandarizados, lo cual arrojó valores entre +2 y -2 validando la estimación de comparaciones múltiples, por ende se optó por aplicar los siguientes intervalos:

$$
\widehat{\Delta}_{j_{1} j_{2}} \mp M \sqrt{C M_{\text {intra }}\left(\frac{1}{n_{j_{1}}}+\frac{1}{n_{j_{2}}}\right)}(7)
$$

Por lo que el proceso aplicado sirvió para poder validar la homogeneidad de los subgrupos.

\section{ANÁLISIS DE LOS RESULTADOS}

La percepción que tienen los alumnos de la carrera Ingeniería de Empresas sobre la calidad es relativamente alta, de tal manera que la tabla 1 indica que en promedio los alumnos están satisfechos en un $83.81 \%$ con el servicio de la carrera desde una perspectiva general. Así mismo, se puede evidenciar una satisfacción docente de un $82.29 \%$, relativamente menor a la perspectiva global de la carrera, sin embargo en cuanto a la percepción de la calidad en los servicios administrativos se puede observar un nivel de 77.54\%; sin embargo es evidente un mayor nivel de dispersión en cuanto a este componente. Para todos los niveles existen respuestas observadas con un nivel de satisfacción de un $100 \%$.

\section{TABLA 1. ESTADÍSTICOS DE PERCEPCIÓN DE LA CALIDAD}

\begin{tabular}{lrrr} 
& SATCARRERA & SATISDOCENTE & SATADMINIS \\
\hline Recuento & 65 & 65 & 65 \\
Promedio & 0,838154 & 0,822857 & 0,775385 \\
Desviación estándar & 0,101595 & 0,107286 & 0,182029 \\
Coeficiente de variación & $12,1213 \%$ & $13,0382 \%$ & $23,476 \%$ \\
Mínimo & 0,52 & 0,485714 & 0,266667 \\
Máximo & 1,0 & 1,0 & 1,0 \\
Rango & 0,48 & 0,514286 & 0,733333 \\
\hline
\end{tabular}

Fuente: los autores.

La tabla 2 indica que en base al error estándar y considerando un nivel de confianza del 95\% se puede asumir que la satisfacción global de la carrera en diversos procesos de muestreo podría estar entre un $81.30 \%$ y un $86.33 \%$; mientras

\begin{tabular}{|c|c|c|c|c|}
\hline & MEDIA & ERROR EST. & $\begin{array}{r}\text { LÍMITE } \\
\text { INFERIOR }\end{array}$ & $\begin{array}{r}\text { LÍMITE } \\
\text { SUPERIOR }\end{array}$ \\
\hline satcarrera. & 0,838154 & 0,0126013 & 0,81298 & 0,863328 \\
\hline satisdocente. & 0,822857 & 0,0133071 & 0,796273 & 0,849441 \\
\hline satadminis. & 0,775385 & 0,0225779 & 0,73028 & 0,820489 \\
\hline
\end{tabular}
que la satisfacción docente podría estar entre un $79.62 \%$ y un $84.94 \%$; y la satisfacción con los servicios administrativos podría situarse entre un $73.03 \%$ y $82.05 \%$.

Fuente: los autores.

Dada la consideración de los niveles de satisfacción por cada componente analizado se procedió a realizar una prueba para poder descartar la no igualdad entre las percepciones sobre los servicios de calidad a nivel de servicios generales de la carrera, servicio docente y servicios administrativos.

TABLA 3. PRUEBA DE KRUSKAL-WALLIS DE DIVERSOS NIVELES

\begin{tabular}{lrr} 
& TAMAÑO DE MUESTRA & RANGO PROMEDIO \\
\hline satcarrera. & 65 & 106,269 \\
\hline satisdocente. & 65 & 98,0923 \\
\hline satadminis. & 65 & 89,6385 \\
\hline
\end{tabular}

Estadístico $=2,84215$ Valor $-\mathrm{P}=0,241454$

Fuente: los autores. 
Una de las preocupaciones en cuanto a la percepción de la calidad en los servicios que brinda la carrera Ingeniería de Empresas era el nivel bajo de satisfacción en cuanto a servicios administrativos desde una perspectiva relativa. Sin embargo, la tabla 3 muestra el estadístico KW considerando un nivel de significación del 5\%. Se infiere que no existe diferencia significativa entre los niveles de satisfacción de la carrera, satisfacción docente y satisfacción en servicios administrativos.

TABLA 4. PRUEBA DE KRUSKaL-WALLIS PARA SATISFACCIÓN GLOBAL DE LA CARRERA

\begin{tabular}{rrr} 
CICLO & TAMAÑ̃O MUESTRA & RANGO PrOMEDI0 \\
\hline 1 & 25 & 33,28 \\
3 & 7 & 39,6429 \\
6 & 12 & 34,5417 \\
7 & 16 & 25,6875 \\
8 & 5 & 42,0 \\
\hline
\end{tabular}

Estadístico $=4,56167$ Valor $-\mathrm{P}=0,335297$

Fuente: los autores.

En cuanto a la satisfacción global de la carrera la tabla 4 indica que no existe diferencia significativa en cuanto a las percepciones por ciclo universitario, es decir que los alumnos de la carrera de Ingeniería de Empresas-Modalidad Dual comparten un criterio de satisfacción de los servicios de la carrera a nivel homogéneo.

TABLA 5. PRUEBA DE KRUSKAL-WALLIS PARA SATISFACCIÓN DOCENTE CICLO TAMAÑO MUESTRA RAMGO PROMEDIO

\begin{tabular}{rrr} 
CICLO & TAMANO MUESTRA & RANGO PROMEDIO \\
\hline 1 & 25 & 31,12 \\
3 & 7 & 35,1429 \\
6 & 12 & 37,0833 \\
7 & 16 & 28,6563 \\
8 & 5 & 43,5
\end{tabular}

Estadístico $=3,3143$ Valor $-\mathrm{P}=0,50667$

Fuente: los autores.

La tabla 5 muestra que la satisfacción con relación a los servicios docentes que tienen los estudiantes de la carrera Ingeniería de Empresas-Modalidad Dual, es homogénea en todos los niveles. En este sentido, la prueba KW refleja un valor $P$ superior al nivel de significancia por lo que se asume que las percepciones sobre los servicios docentes son iguales en cada ciclo.
TABLA 6. PRUEBA DE KRUSKAL-WALLIS PARA SATISFACCIÓN SERVICIOS ADMINISTRATIVOS

\begin{tabular}{rrr} 
CICLO & TAMAÑO MUESTRA & RANGO PROMEDIO \\
1 & 25 & 32,24 \\
3 & 7 & 47,0 \\
6 & 12 & 33,0833 \\
7 & 16 & 25,4063 \\
8 & 5 & 41,3 \\
\hline
\end{tabular}

Estadístico $=7,57432$ Valor $-\mathrm{P}=0,108477$

Fuente: los autores.

En cuanto a los servicios administrativos, la tabla 6 indica que no hay diferencia significativa entre las percepciones de los servicios administrativos, a nivel de ciclos lectivos dado que el valor $P$ del coeficiente de KW es mayor que el nivel de significancia de 0.05 . Sin embargo la validación de la igualdad para la percepción en la calidad de los servicios administrativos no fue rectificada por la prueba de múltiples rangos.

tABLA 7. PRUEBAS DE MÚlTIPLE RANGoS PARA SATISFACCIÓN ADMINISTRATIVA (MÉTODO: 95,0 PORCENTAJES LSD)

\begin{tabular}{rrrr} 
CICLO & CASOS & MEDIA & $\begin{array}{r}\text { GRUPOS } \\
\text { HOMOGÉNEOS }\end{array}$ \\
7 & 16 & 0,7 & $\mathrm{X}$ \\
6 & 12 & 0,766667 & $\mathrm{XX}$ \\
1 & 25 & 0,773333 & $\mathrm{XX}$ \\
8 & 5 & 0,866667 & $\mathrm{XX}$ \\
3 & 7 & 0,904762 & $\mathrm{X}$ \\
\hline
\end{tabular}

Fuente: los autores.

El resultado del análisis mostrado en la tabla 7 evidenció que por lo menos existen cuatro grupos homogéneos identificados en el análisis. El primer grupo lo conforman los alumnos del séptimo, sexto, primer y octavo ciclos y él otro grupo lo conforman los de tercer ciclo.

\section{DISCUSIÓN}

La metodología aplicada en el proceso generó robustez en los resultados y permitió determinar de una manera más específica cual es la real percepción de la calidad medida en base a aspectos que consideran prioritarios los estudiantes quienes realmente son los perceptores del servicio. Según los resultados obtenidos, la percepción de la calidad de la carrera por parte de los estudiantes fue medida bajo tres factores: el primero, satisfacción con la carrera en tér- 
minos generales determinado por la formación académica recibida; el segundo factor es la satisfacción de la calidad de los docentes, dado por el nivel de conocimientos de los docentes y la relación con los estudiantes; y el tercer factor considerado fue la satisfacción con la calidad de la administración de la carrera, enfocado a la atención brindada por el área académico.

Las dimensiones anteriores no convergen con los usadas por Ruano (2002). Las aplicadas son: los factores propios de la organización de la docencia, factores de proceso de renovación curricular y formación académica con el desempeño profesional. Ruano plantea que estos tres niveles son susceptibles de producir indicadores de la calidad de la educación que deben adaptarse a la situación educativa concreta.

Mientras que en su estudio sobre la determinación de la percepción de la calidad de servicio en una universidad, Ríos (2005), aplicó tres factores: el primero, llamado de contactos personales, caracterizado por la capacidad y disposición de las personas para proveer el servicio; el segundo factor es la empatía, dado por la necesidad de comprensión de necesidades y atención personalizada por parte de los clientes del servicio; y el tercer factor, elementos tangibles, formado por las instalaciones y equipos disponibles para proveer el servicio; siendo el principal de estos tres factores, el correspondiente a los elementos tangibles.

Por lo tanto, se recomienda realizar investigaciones de homogeneidad basadas en pruebas no paramétricas incluyendo estándares oficiales que validen la calidad de los servicios educativos, para de esta manera poder aportar a la validez externa de la investigación.

CONCLUSIONES

La investigación permitió inferir que existe igualdad entre los niveles de satisfacción vinculados a tres áreas críticas: (a) carrera en general, (b) aspectos administrativos, y (c) docentes. Así mismo se confirmó que todos los alumnos tienen una percepción homogénea en cuanto a la calidad en los servicios que brinda la carrera, todo desde una perspectiva global. Así mismo se muestra que existe una satisfacción homogénea entre todos los niveles lectivos con relación a la percepción de la calidad docente. Otro factor importante analizado fueron los servicios administrativos que también demostraron un nivel de homogeneidad. Sin embargo, el valor $P$ fue más estrecho, es decir que este podría ser más sensible a variación en los niveles de significancia, por tanto se demostró la posible existencia de dos grupos distintos en cuanto a percepciones de calidad.

\section{REFERENCIAS BIBLIOGRÁFICAS}

Araya, I. (2008). La formación dual y su fundamentación curricular. Revistas Académicas, Universidad de Costa Rica Vol.32 Núm.1, 1.

Coromina Rovira, E., Tesouro , M., Capell Castañer, D., Teixidó Saballs, J., Pèlach Busom, J., y Cortada, R. (2006). Percepciones del profesorado ante la incorporación de las competencias genéricas en la formación universitaria. Revista de Educación, 341 (14), 301-336.

De la Fuente, J., Martínez, J., Peralta, F., \& García, A. (2010). Percepción del proceso de enseñanzaaprendizaje y rendimiento académico en diferentes contextos instruccionales de la Educación Superior. Psicothema, 22 (4), 1-7.

Dieter, E. (2013). El sistema dual en Alemania. ¿Es posible transferirlo al extranjero? Fundacion Bertlsmann, $82,4-72$.

Garbanzo, G. (2007). Factores asociados al rendimiento académico en estudiantes universitarios, una reflexion desde la calidad de la educacion superior publica. Revista Educación, 31 (1), 43-63.

Hernández, D. (2002). Estrategias docentes para un aprendizaje significativo. Mexico: Mc Graw Hill.

Herrera, L., y Lorenzo - Quiles, O. (2009). Estrategias de aprendizaje en estudiantes universitarios. Universidad de Granada, 12 (3), 75-98.

Hierro, G. (1994). Naturaleza y Fines de la Educación Superior. Mexico: UNAM.

Hinojo, F., Aznar, I., \& Caceres, M. (2009). Percepciones del alumnado sobre el blended learning en la Universidad, Revista cientifica de Educomunicacion. 33 (17), 165-174.

Kruskal, W., \& Wallis, W. A. (1952). Use of Ranks in one criterion Variance Analysis. Journal of the American Statistical Association, 47(260), 584-618.

Lema Soto, L., Salazar Torres, I. C., Varela Arévalo, M. T., Tamayo, J., Rubio, A., y Botero, A. (2009). Comportamiento y salud de los jovenes universitarios: Satisfaccion con el estilo de vida. Cali (Colombia:)Pontificia Universidad Javeriana.

Lind, D., Marchal, W., y Wathen, S. (2012). Estadística aplicada a los negocios ya la economía. New York: McGraw-Hill.

Mauri, T., Coll, C., y Onrubia, J. (2004). La evaluación de la calidad de los procesos de innovación. Grupo de Innovación Docente en Psicología de la Educación, Revista de Docencia Universitaria, 5 No (1), 1-11. 
Merida, R. (2006). Nueva percepción de la identidad profesional. Revista Electrónica de Investigación Educativa, 8 (1), 1-5.

Ríos, D. (2005). Validación de una escala de medida para la determinación de la calidad de servicio en una institucion de educacion superior. Flumen, 1 (1), 2-10.

Ruano, C. (2002). Más allá de la evaluación por resultados: Planteamientos metodológicos en torno al proceso de autoanálisis institucional y la construcción de indicadores de la calidad educativa en el contexto universitario. OEIRevista Iberoamericana de Educación, 1-9.
Salinas, J. (2004). Innovación Docente y Uso de las TIC en la enseñanza universitaria. Universidad y Sociedad del Conocimiento, 1 (1), 1 -16.

Vega. L. (2005). Evaluación Programa en Administración de Oficinas. Heredia: Universidad Nacional, Escuela de Secretariado Profesional. 